\title{
Low-Dose Cyproterone Acetate Treatment of Sexual Acting Out in Men With Dementia
}

\author{
Peter Haussermann, David Goecker, Klaus Beier, and Stefan Schroeder
}

ABSTRACT. Increased sexual activity in patients with dementia is problematic for caregivers and has no proven treatment. This report describes two male patients who had dementia with pronounced sexual acting out. One patient had vascular dementia and the other patient had Parkinson's disease with associated dementia. After nonresponse to neuroleptics and sedatives, low-dose cyproterone acetate successfully reduced sexual acting out without relevant side effects in both patients.

KEYWORDS: Sexual acting out; dementia; antiandrogen; cyproterone acetate

Different disciplines, from psychiatry to general medicine, are nowadays faced with treating dementia patients. Neuropsychiatric symptoms such as depression, hallucinations, and agitated behavior contribute most to carers' burden (Assal \& Cummings, 2002; Krueger $\&$ Kaplan, 2000). The frequency of disruptive sexual behavior in dementia patients has been shown to vary from $4 \%$ to $14 \%$ (Kuhn et al., 1998). Increased sexual activity has been reported in patients with epilepsia, Parkinson's disease (Beier et al., 2000; Vogel \& Schiffter, 1983), traumatic brain injury (Miller et al., 1986), multiple sclerosis, and stroke (Monga et al., 1986). Sexual acting out has been demonstrated to be related to

From the Department of Neurology, TU Munich, Munich, Germany (P. Haussermann), Department of Psychiatry, Ruhr University Bochum (D. Goecker; and S. Schroeder), and Humboldt University, Berlin, Germany (K. Beier). temporal and frontal lobe involvement (Absher et al., 2000) as well as to basal ganglia (Absher et al., 2000), cingulate cortex, or amygdaloid nucleus lesions (Absher et al., 2000; Monga et al., 1986).

Sexual acting out may be manifested by an increased libido or sexual disinhibition (Miller et al., 1986; Rosler \& Witztum, 2000). In dementia, it has to be regarded as beyond the person's understanding and control, not as a personal preference or willful behavior. There is no proven treatment (Miller et al., 1986), although reports have described reduction of increased sexual activity using pharmacotherapy and behavioral/environmental (see Table 1) interventions (Cooper, 1987; Cooper et al., 1972; Levitsky \& Owens,

Offprints. Requests for offprints should be directed to Dr. Peter Haussermann, Department of Neurology, Klinikum rechts der Isar, TU München, Moehlstr. 28, 81657 München, Germany. email: peter.haeussermann@gmx.de 
1999). Putative antiandrogen antilibidinal medications include cyproterone acetate (CPA), flutamide, medroxyprogesterone acetate (MPA), GnRH-analogues, estrogen, spironolactone, ketoconazole, and cimetidine (Lardinois \& Mazzaferri, 1985; Rosler \& Witztum, 2000; Wiseman et al., 2000). Serotonergic substances have been shown to decrease delusions and irritability associated with hypersexual behavior (Stein et al., 1992; Stewart \& Shin, 1997).

\section{PATIENTS AND METHODS}

A systematic study of the treatment of increased sexual activity in patients with dementia has not been reported. Therefore, our purpose was to evaluate the impact of CPA, a hormonal hydroxyprogesterone derivative with a competitive antagonism in the androgen receptor in patients with dementia that exhibited sexual acting out. Caregivers and doctors recorded sexual behaviors and determined the degree of improvement after antiandrogen therapy. Caregivers consented to CPA treatment. Severity of dementia was scored using the MiniMental State Examination (MMSE).

\section{Case A}

Patient A, a 79-year-old male retired entrepreneur, has had a bipolar affective disorder since 1970, akinetic-rigid Parkinson's disease since 1984, and slowly progressive cognitive deficits since 1989. A computed tomographic (CT) scan showed slight cortical brain atrophy in 1989. Initially, mainly his short-term memory was affected. Cognitive status further deteriorated and Parkinson's disease-associated dementia was diagnosed in 1998, when the patient scored 22/30 on the MMSE and was impaired both in activities of daily living as well as in social functioning.

On admission in 2000, his wife reported (auto)-sexual activity over several months. On the gerontopsychiatric ward, he masturbated in front of female patients and nursing staff several times a week. No clear association to a manic episode could be found. There were also verbal components with sexual intent. In his MMSE on admission, he scored 19 out of 30 points. His medication consisted of valproate $(1,500 \mathrm{mg} / \mathrm{day})$, olanzapine ( $7.5 \mathrm{mg} /$ day), lithium ( $800 \mathrm{mg} /$ day), lorazepam ( $1 \mathrm{mg} /$ day), and L-Dopa (250 $\mathrm{mg} /$ day). Masturbation in public dramatically diminished within a few days when he was placed on $10 \mathrm{mg}$ CPA per day. A dose reduction of CPA to $5 \mathrm{mg}$ per day was twice followed by a relapse of sexual acting out. After the dosage was reincreased to $10 \mathrm{mg}$ per day, the symptoms of agitated sexual behavior disappeared.

\section{Case B}

Patient B, a 74-year-old male retired business consultant, was admitted to the psychogeriatric ward because of delusional misidentifications, visual and acoustic hallucinations, increased sexual appetence towards his wife, and compulsive masturbation with ejaculations in public and private places.

Four years prior to admission, he had had several transitory ischemic attacks. The patient had a long-standing history of arterial hypertension with bad control of blood pressure. Further medical conditions included type II diabetes mellitus treated with insulin and cardiac arrhythmia with intermittent atrial fibrillation and sick sinus syndrome. Therefore, the patient received a pacemaker. A CT scan 
TABLE 1. Putative Treatment Approaches for Sexual Acting Out in Patients With Dementia
1. Nonpharmacological
- Environmental interventions
- Nursing strategies
- Psychotherapy
2. "Mood stabilizers" - Valproate $300-600 \mathrm{mg} /$ day
- Carbamazepine 200-600 mg/day
3. Antidepressants
- SSRIs: e.g., paroxetine 20 mg/day (Leo \& Kim, 1995), fluoxetine $10 \mathrm{mg} /$ day
- Tricyclic antidepressants: clomipramine 75-150 mg/day (Leo \& Kim, 1995)
4. Neuroleptics
- Quetiapine $50-100 \mathrm{mg} / \mathrm{day}$
- Risperidone $0.5-1 \mathrm{mg} /$ day
- Melperone $50-100 \mathrm{mg} / \mathrm{day}$
- Olanzepine 5-10 mg/day

5. Antiandrogens

\section{Hormonal antiandrogens}

- Cyproterone acetate 10-300 mg/day (Cooper, 1987; Levitsky \& Owens, 1999)

- Spironolactone $75 \mathrm{mg} /$ day (Wiseman et al., 2000)

Nonhormonal antiandrogens

- Medroxyprogesterone acetate 200-300 mg/week intramuscularly (Cooper, 1987)

- Cimetidine 600-1,600 mg/day (Wiseman et al., 2000)

- Ketoconazole 100-200 mg/day (Wiseman et al., 2000)

6. Estrogens - Estradiol $2 \mathrm{mg} /$ day (Flynn, 1999)

- Conjugated estrogens $1.25 \mathrm{mg} /$ day (Flynn, 1999)

7. GnRH analogues

- Leuprolide $11.25 \mathrm{mg}$ every 3 months subcutaneously (Levitsky \& Owens, 1999)

Note. SSRIs = selective serotonin reuptake inhibitors.

performed at that time showed moderate periventricular leucoaraiosis but no lacunar infarcts.

Three months prior to hospital admission, he had a severe head trauma after a car accident with consecutive intracerebral hemorrhage in the right frontal lobe and right-sided subdural hematoma. According to his wife, there was a continuous cognitive decline over the preceding years but no inappropriate sexual behavior.

On admission, his MMSE score was $8 / 30$. There were no frontal release signs or changes in dietary habits. A CT scan showed periventricular leucoaraiosis, global brain atrophy, and lacunar infarcts in both temporal lobes and the left occipital cortex.

Psychopharmacotherapy consisted of valproate ( $300 \mathrm{mg} /$ day), mirtazepine ( 45 $\mathrm{mg} /$ day), and risperidone (1 $\mathrm{mg} /$ day). After 1 week in the hospital, we started the application of $10 \mathrm{mg} \mathrm{CPA} /$ day and sexually inappropriate behavior disappeared within a few days. Again, a dose reduction of CPA to $5 \mathrm{mg}$ per day was followed by a relapse of sexual acting out. 
After the dosage was reincreased to 10 mg per day, the symptoms of sexual acting out disappeared. The patient was followed up for 6 months and there was no relevant change in his poor cognitive status. On his MMSE 9 months after the accident, he scored $8 / 30$ points.

The patient was diagnosed as having vascular dementia after traumatic intracerebral hemorrhage. Neuroimaging showed periventricular leucoaraiosis as well as multiple lacunar infarcts and brain atrophy. Dementia diagnosis was based on neuroimaging findings, both impairment in his activities and experiences of daily living (personal hygiene, dressing, eating) as well as impaired social functioning. Formal neuropsychological testing was difficult to perform because of severe attention deficits and lack of cooperation. Tower of Hanoi testing was not possible; neither was the use of other standardized neuropsychological tests evaluating frontal lobe function. There was reduced attentional span, and cognitive speed was severely impaired. Both short-term memory and long-term memory were deficient.

\section{DISCUSSION}

It should be noted that in Patient A, increased sexual activity interpreted as a complication of dopaminergic therapy in Parkinson's disease seems improbable (Beier et al., 2000), though it cannot be fully excluded (Vogel \& Schiffter, 1983). Head trauma with frontal lobe hemorrhage may at least partially account for increased sexual activity seen in Patient B (Miller et al., 1986; Monga et al., 1986), even in the absence of frontal release signs or dietary changes.
Our two cases suggest that CPA given in a low dose-less than a tenth of the usually recommended dose-may be useful to reduce sexually inappropriate behavior in men with dementia. For the treatment of paraphilias (Rosler \& Witztum, 2000) in younger patients, antiandrogen therapy may include MPA and CPA. MPA can be administered at 300 mg per week intramuscularly, while CPA can be given in daily oral doses between 50 and $100 \mathrm{mg}$ or $300 \mathrm{mg}$ intramuscularly weekly (Briken et al., 2000).

The only data supporting the use of antiandrogen therapy for the treatment of hypersexuality or paraphilias in older patients with dementia are in the form of case reports. The patients were administered MPA $300 \mathrm{mg}$ per week intramuscularly for 1 year. Alternatively, 10 to $100 \mathrm{mg}$ of CPA can be given daily (Cooper, 1987; Levitsky \& Owens, 1999), depending on tolerability and side effects.

The mechanisms underlying response to low-dose CPA in these two dementia patients are difficult to elucidate. Major factors might be the known lower testosterone blood levels in elderly patients and pharmacokinetic changes associated with aging, i.e., longer plasma half-life and relatively higher CPA levels (Krueger \& Kaplan, 2000; Levitsky \& Owens, 1999). Pharmacodynamic changes with increased receptor sensitivity may as well be important in elderly (dementia) patients (Bradford, 2001). Neurotransmitter changes within hypothalamic and frontal loops involved in the control of libido and sexual activity that occur in normal aging as well as in the course of dementia may also contribute to lowdose CPA response seen in both patients (Bradford, 2001). 
Our observation has limitations, including a potential recall bias by the patient's caregivers during follow-up, and the subjective nature of responses. We would like to emphasize the putative ethical and legal implications of CPA pharmacotherapy, mainly concerning the inability of dementia patients to consent to pharmacotherapy and the possible side effects, including liver dysfunction, carcinogenic effects, osteoporosis, depression, or gynecomastia. CPA may also be used for the treatment of female hypersexuality (Mellor et al., 1988).

In conclusion, the prescription of CPA was helpful in the treatment of our patients who demonstrated sexual acting out. After nonresponse to conventional psychopharmacotherapy (sedatives, neuroleptics, and valproate), the prescription of low-dose CPA reduced sexual acting out significantly in both patients without relevant side effects. Subjectively, intensity of increased sexual activity in both patients decreased to the point that it was no longer considered problematic by the caregivers. Objectively, the frequency of (auto)-sexual activity decreased significantly.

Nevertheless, there are no systematic studies available today concerning the treatment of inappropriate sexual behavior in dementia. Prospective double-blind studies that clearly define response variables and focus on longterm side effects are needed to confirm our observations.

\section{REFERENCES}

Absher, J. R., Vogt, B. A., Clark, D. G., Flowers, D. L., Gorman, D. G., et al. (2000). Hypersexuality and hemiballism due to subthalamic infarction. Neuropsychiatry, Neuropsychology, and Behavioral Neurology, 13, 220-229.
Assal, F., \& Cummings, J. L. (2002). Neuropsychiatric symptoms in the dementias. Current Opinion in Neurology, 15, 445-450.

Beier, K. M., Lüders, M., \& Boxdorfer, S. A. (2000). Sexualität und Partnerschaft bei Morbus Parkinson [Sexuality and partnership in patients with Parkinson's disease]. Fortschritte der Neurologie-Psychiatrie, 68, $564-575$.

Bradford, J. M. (2001). The neurobiology, neuropharmacology, and pharmacological treatment of the paraphilias and compulsive sexual behaviour. Canadian Journal of Psychiatry, 46, 26-34.

Briken, P., Berner, W., Noldus, J., Nika, E., \& Michl, U. (2000). Therapie mit dem LHRH-Agonisten Leuprolinacetat bei Paraphilien und sexuell aggressiven Impulshandlungen [Therapy of paraphilias and sexual acting out with the LHRH agonist leuprolinacetate]. Der Nervenarzt, 71, 380-385.

Cooper, A. J. (1987). Medroxyprogesterone acetate (MPA) treatment of sexual acting out in men suffering from dementia. Journal of Clinical Psychiatry, 48, 368-370.

Cooper, A. J., Ismail, A. A., Phanjoo, A. L., \& Love, D. L. (1972). Antiandrogen (cyproterone acetate) therapy in deviant hypersexuality. British Journal of Psychiatry, 120, 59-63.

Flynn, B. L. (1999). Pharmacologic management of Alzheimer disease. Part I: Hormonal and emerging investigational drug therapies. Annals of Pharmacotherapy, 33, 178-187.

Krueger, R. B., \& Kaplan, M. S. (2000). Disorders of sexual impulse control in neuropsychiatric conditions. Seminars in Clinical Neuropsychiatry, 5, 266-274.

Kuhn, D. R., Greiner, D., \& Arseneau, L. (1998). Addressing hypersexuality in Alzheimer's disease. Journal of Gerontological Nursing, $4,44-50$.

Lardinois, C. K., \& Mazzaferri, C. L. (1985). Cimetidine blocks testosterone synthesis. Archives of Internal Medicine, 145, 920-922.

Leo, R. J., \& Kim, K. J. (1995). Clomipramine treatment of paraphilias in elderly 
demented patients. Journal of Geriatric Psychiatry and Neurology, 8, 123-124.

Levitsky, A. M., \& Owens, N. J. (1999). Pharmacologic treatment of hypersexuality and paraphilias in nursing home residents. Journal of the American Geriatrics Society, 47, 231-234.

Mellor, C. S., Farid, N. R., \& Craig, D. F. (1988). Female hypersexuality treated with cyproterone acetate. American Journal of Psychiatry, 145, 1037.

Miller, B. L., Cummings, J. L., McIntyre, H., Ebers, G., \& Grode, M. (1986). Hypersexuality or altered sexual preference following brain injury. Journal of Neurology, Neurosurgery, and Psychiatry, 49, 867873.

Monga, T. N., Monga, M., Raina, M. S., \& Hardjasudarma, M. (1986). Hypersexuality in stroke. Archives of Physical Medicine and Rehabilitation, 67, 415-417.
Rosler, A., \& Witztum, E. (2000). Pharmacotherapy of paraphilias in the next millennium. Behavioral Sciences \& the Law, $18,43-56$.

Stein, D., Hollander, E., Anthony, D., et al. (1992). Serotonergic medications for sexual obsessions, sexual addictions and paraphilias. Journal of Clinical Psychiatry, 53, 267-271.

Stewart, J. T., \& Shin, K. J. (1997). Paroxetine treatment of sexual disinhibition in dementia. American Journal of Psychiatry, $154,1474$.

Vogel, H. P., \& Schiffter, R. (1983). Hypersexuality-A complication of dopaminergic therapy in Parkinson's disease. Pharmacopsychiatry, 16, 107-111.

Wiseman, S. V., McAuley, J. W., Freidenberg, G. R., \& Freidenberg, D. L. (2000). Hypersexuality in patients with dementia: Possible response to cimetidine. Neurology, 54, 2024. 